\title{
Oculomotor rehabilitation in children with dyslexia
}

\author{
Fatemeh Jafarlou ${ }^{1}$, Farnoush Jarollahi*1, Mohsen Ahadi ${ }^{1}$, Vahid Sadeghi-Firoozabadi ${ }^{2}$, \\ Hamid Haghani ${ }^{3}$
}

Received: 7 Aug 2017

Published: 24 Dec 2017

\begin{abstract}
Background: Dyslexia is the most common learning disorder. Visual and oculomotor deficits in dyslexic children have been reported. The purpose of this study was to measure oculomotor parameters and analyze the effect of oculomotor rehabilitation strategies on dyslexia.

Methods: Binocular eye movements were recorded by oculomotor subtype of videonystagmography (VNG) testing on 30 children with dyslexia and 20 typical reader children (aged 8-12) in both genders. Dyslexic children were diagnosed with DSM-V scale by experts in reading disorder centers. We studied those children with developmental dyslexia, who had deficits in eye movements recording. Dyslexic children were divided into 2 groups of case and control. Oculomotor rehabilitation (including fixation, saccade, and tracking training) was performed in case group for 1 hour, twice weekly for 8 weeks. Before the intervention, results of oculomotor tests were compared between 3 groups (healthy, case, and control). Then, to analyze the effect of the intervention, results of oculomotor tests were compared between case and control groups in pre- and post- intervention stage. Data were analyzed by independent and paired samples $t$ tests, ANOVA, and repeated measures tests in SPSS v. 21.

Results: There were significant differences in oculomotor characteristics of dyslexic children in comparison with those reported in typical children. Oculomotor rehabilitation intervention had a positive effect on improvement of oculomotor responses and eye movements in dyslexic children. Moreover, there was no statistically significant difference between dyslexic children and non-dyslexic children in oculomotor skills after the training.

Conclusion: Our results showed the positive effects of oculomotor rehabilitation on eye movements. Primary oculomotor assessment in dyslexic children and early use of oculomotor rehabilitation combined with other treatments are highly recommended.
\end{abstract}

Keywords: Dyslexia, Eye movements, Oculomotor deficiency, Oculomotor rehabilitation

Copyright@ Iran University of Medical Sciences

Cite this article as: JafarlouF, Jarollahi F, Ahadi M, Sadeghi-Firoozabadi V, Haghani H. Oculomotor rehabilitation in children with dyslexia. Med $J$ Islam Repub Iran. 2017(24 Dec);31:125. https://doi.org/10.14196/mjiri.31.125

\section{Introduction}

Reading is one of the important activities of daily life, which requires precise coordination of both lower level oculomotor processes (version, accommodation, and vergence) and higher-level non- oculomotor processes (e.g., attention, language, cognition, and memory)(1). Dyslexia is a specific developmental disorder in fluent reading acquisition, reading comprehension, and spelling skills that is not a direct consequence of distribution in total intelligence, specific neurologic disorders, uncorrected visual or auditory problems, psychological problems, or academic difficulties. Dyslexia affects at least $5 \%$ of school age children and is more prevalent in boys than in girls (2). This disorder has a neurological basis, moreover, genetic factors also affect ability to read and spell(2). Based on the onset of the disorder, dyslexia is divided into 2 types: developmental dyslexia, and acquired dyslexia. In developmental dyslexia, phonological disorder is inherited in the cortex (3). Acquired dyslexia is caused by trauma or injury to the part of the brain that controls reading and writing skills, and at older ages, the disorder can be caused by a tumor or stroke (4).

The precise etiology of dyslexia is unknown. Although one of the most accepted theories about the causes of dyslexia suggests that the main defect is in phonological $\uparrow$ What is "already known" in this topic:

Dyslexic children have abnormal eye movements during reading, and oculomotor tests are well known tools for examining eye movements in this population.

$\rightarrow$ What this article adds:

Oculomotor rehabilitation is an effective treatment protocol for improving eye movements in children with dyslexia and improves reading indirectly. 
awareness and poor phonological encoding, butother theories are still open for discussion. For example, there is persuasive evidence of visual and oculomotor abnormalities in dyslexia (1-12). The prevailing but also controversial theory in this context is the Magnocellular-Dorsal (MD) theory (7-10). The visual magnocellular system plays an important role in guiding attention, avoiding dark and faded images, and fixing eyes on the target, and it is responsible for timing visual events during reading(12). Some studies have declared that visual magnocellular system of the lateral geniculate nucleus is immature in dyslexia and appears as impaired eye movement patterns and word recognition $(13,14)$. Oculomotor impairment in dyslexia causes high-level attentional aspects to be allocated to basic oculomotor activities during reading (such as an alternate focus on words) and reduces the ability of the individual in understanding words (1). In short, the old dominant view, which tries to describe dyslexia as a result of a single cause, is still controversial, and it is apparent that dyslexia is a highly complex disorder that is better described using a multifactorial and probabilistic model (15).

Auditory, phonological, and linguistic treatments that are often being used for treatment of dyslexia, affect high levels of the nervous system and postlexical pathways (27-30).However, recent studies have shown that brainstem dysfunction may cause reading problems (31-35).
Therefore, to assess online reading processes and evaluate and improve the performance of lower levels of the cortex in the brain stem, it seems that eye movements recording during reading and oculomotor rehabilitation are valuable procedures (36-38). There are several studies on the recording of eye movements to diagnose dyslexia disorders (15-25). However, in the treatment area of dyslexia using oculomotor rehabilitation, there are few studies that have evaluated its effect on individuals with acquired dyslexia $(1,14,39-42)$.There are no studies that directly address the effectiveness of oculomotor rehabilitation in developmental dyslexia. Therefore, the present study aimed at evaluating the impact of oculomotor rehabilitation on improving eye movements in children with dyslexia. In this study, it was assumed that stimulus presentation as searching and tracking an object via oculomotor rehabilitation can improve visual tracking and visual attention, which are both impaired in individuals with learning disorder, especially in dyslexia.

\section{Methods}

The study protocol required a consecutive 11-week commitment by the dyslexic group. Figure 1 displays the flowchart of the study design. In general, we conducted this study in 2 phases. In the first phase, we aimed at finding the oculomotor response difference between the dyslexic and non- dyslexic children. Therefore, we used ocu-




lomotor tests to find these differences. In the second phase, we used oculomotor rehabilitation intervention to treat a subgroup of dyslexic children. In this phase, oculomotor tests were again taken from the 2 groups to analyze the effectiveness of the intervention.

Participants: A total of 50children aged 8 to 12 years, including 20 non-dyslexic children (mean age: $9.55 \pm 0.94$ years; 6 females and 14 males) and 30 children with developmental dyslexia (mean age 9.1 \pm 1.24 years; 6 females and 24 males) participated in this study. The number of males was 3 times more than that of the females $(76 \%$ vs. $24 \%$ ), this ratio remained approximately the same after distribution of the population into 3 groups. We apportioned dyslexic children into two 15-member groups: (1) dyslexic children with rehabilitation intervention (case group; mean age $8.94 \pm 1.03$ years; 4 females and 11 males), and (2) dyslexic children without rehabilitation intervention (control group; mean age 9.27 \pm 1.44 years; 2 females and 13 males). Participants were selected based on inclusion and exclusion criteria (Table 1). The sample size was calculated, with the power of $80 \%$ and type I error of $5 \%(\alpha=0.05)$, based on the following formula:

$$
n=\left(\frac{\left(z_{1-\frac{\alpha}{2}}+z_{1-\beta}\right) \delta}{d}\right)^{2}
$$

and according to the results of the pilot study and literature review. In the pilot study, eye movements were recorded in 5 dyslexic and 5 non-dyslexic children. Then, the mean and standard deviation values of the 2 oculomotor tests including saccades and tracking were calculated, and the highest standard deviation was placed in the formula. After calculations by expert statisticians, the numbers of 20 persons in the healthy group and 30 in the dyslexiagroup were obtained. We selected dyslexic children from 3 specific schools for learning disability children. Dyslexic children were diagnosed based on DSM-V criteria and according to experts' opinion at centers of learning disabilities. The specialized team, who diagnosed dyslexic children in these centers, included a psychiatrist, a clinical psychologist, a health psychologist, one exceptional children psychologist, one educational psychologist, a cognitive psychologist, and a counselor and psychologist for children with special needs. Mistakes of dyslexic children based on DSM-V are usually characterized by such symptoms as deletion, addition, or reversal of the words. All children enrolled in the study had an intelligence quotient of $>80$ on the standard IQ tests recorded in their health files. Non-dyslexic children were selected from elementary students in traditional schools in Tehran. Both groups of dyslexic and non-dyslexic children were selected from the same areas in Tehran (municipal zone 3 of Tehran), so that the children did not differ significantly in cultural, educational, and economic characteristics. In addition, in the demographic questionnaires that were completed by parents, we asked about their income and education level. Random sampling method was used to select a sample of each group. The sample was selected randomly by referring to learning disabilities centers and elementary schools in Tehran. This study was approved by the Ethics Committee of Iran University of Medical Sciences (code number: IR.IUMS.REC.1394.9211303210).

\section{Study design}

Evaluative procedures: To begin the study process, we explained the process and duration of the study to the parents and obtained their written consent. Case history and basic auditory assessment (including audiometry and tympanometry tests) were performed to ensure the health of the middle ear and hearing sensitivity. Eye movements were evaluated by oculomotor tests (including gaze, tracking, saccades, and optokinetic tests) and spontaneous nystagmus test in both dyslexic and non-dyslexic children. Oculomotor test was used to evaluate the central vestibular pathway. These tests were taken using the videonystagmography (VNG) instrument (Interacoustics VO425-2D-VOGfw model). In oculomotor test, a binocular infrared goggle was used to record eye movements. Dyslexic children were randomly divided into 2 equal case and control groups ( $\mathrm{n}=15$ in each group). We provided oculomotor rehabilitation exercises to the case group in addition to constant conventional treatment, which was given in the learning disorders center; the control group did not receive oculomotor rehabilitation intervention. At the end of oculomotor rehabilitation, oculomotor tests were again taken from both groups of dyslexic and non-dyslexic children to compare the results of the tests; and finally, the impact of rehabili-

\begin{tabular}{|c|c|}
\hline $\begin{array}{l}\text { Inclusion } \\
\text { criteria }\end{array}$ & $\begin{array}{l}\text { - Aged } 8 \text { to } 12 \text { years } \\
\text { - Diagnose of dyslexia based on the reading test and opinion of experts } \\
\text { - Deficits in the eye movements in dyslexic children } \\
\text { - Normal hearing in both ears (hearing threshold less than } 25 \mathrm{~dB} H \mathrm{HL} \text { in } 250 \text { to } 8000 \mathrm{~Hz}) \\
\text { - Normal or corrected vision } \\
\text { - Normal intelligence }(>80) \text { using Wechsler IQ test results-modified for children, available in the medical rec- } \\
\text { - } \text { ords } \\
\text { - } \text { Ronolingualism and proficiency in the Persian language as a native language }\end{array}$ \\
\hline $\begin{array}{l}\text { Exclusion } \\
\text { criteria }\end{array}$ & $\begin{array}{l}\text { - Necessary use of ritalin and other drugs during testing and rehabilitation in dyslexic children } \\
\text { - Suffering from associated neurologic disorders, according to parents and medical reports } \\
\text { - History of ear diseases, head injury or accident, brain surgery, and/or epilepsy, according to parents and medi- } \\
\text { - cal reports }\end{array}$ \\
\hline
\end{tabular}




\begin{tabular}{ll} 
Table 2.Oculomotor tests description \\
\hline Oculomotor tests & Explanation of tests \\
Gaze test & $\begin{array}{l}\text { The child will be asked to sit on a chair at a distance of one meter from a big monitor TV. Then, the child was } \\
\text { asked to simply look at the dots at center, } 30 \text { degrees to right and left, without head movement. Presence or ab- } \\
\text { sence of nystagmus was analyzed. }\end{array}$ \\
Tracking test & $\begin{array}{l}\text { For tracking test, the child was asked to slowly track the dot moving at a speed of } 2.0 \mathrm{dg} / \mathrm{s} \text { to different sides with- } \\
\text { out moving his/her head. Gain and symmetry of response on both sides were analyzed. }\end{array}$ \\
Saccade test & $\begin{array}{l}\text { Saccades are the fastest eye movements to locate the target on the retina. In this test, the child was asked to simply } \\
\text { look at dots moving with a velocity of 5-30 randomly to different sides. Latency, velocity and accuracy parameters } \\
\text { were analyzed. } \\
\text { Optokinetic is the ability of a person to maintain visual fixation when he/she or part of the environment is moving. } \\
\text { In this test, the colored stripes move at speeds of } 20,35, \text { and } 50 \text { dg/s to left and right. The child was asked to look at } \\
\text { the middle and count the number of strips without moving the head. Gain and symmetry of response on both sides } \\
\text { were analyzed. }\end{array}$ \\
\hline
\end{tabular}

tation on improving the oculomotor skills was evaluated by appropriate statistical tests.

Quantitative variables of this study were tracking gain (in percent), optokinetic gain (in percent), saccade latency (milliseconds), saccade velocity (degrees per second), and saccade accuracy (in percent). Qualitative variables were presence or absence of spontaneous nystagmus and gaze nystagmus and symmetry/ dissymmetry of tracking and optokinetic responses. More details of oculomotor tests are presented in Table 2. Tracking gain refers to the speed of eye movements relative to the speed of the moving target; tracking symmetry means symmetry of response between eyes; and saccade latency defines the distance between the creation of target and the start of responding. The speed of eye movements during a saccade is defined as saccade velocity; saccade accuracy means the precision of eye movements during a saccade for placement of target on the retina; optokinetic gain means the maximum speed of eye movement divided by the speed of moving targets; and finally, optokinetic symmetry means symmetry of response between eyes (43).

Treatment protocol: In the present study, due to lack of oculomotor norm data for children, oculomotor results in non-dyslexic children were considered as a measure for the diagnosis of poor responses in dyslexic group. Dyslexic children with poor response in one or both tests of saccades and tracking compared to non-dyslexic children were recognized and entered at the second stage of the study. Then, half of them, as a case group, received 16 one-hour sessions of individually monitored oculomotor rehabilitation over 2 months. Oculomotor rehabilitation includes accommodative exercises focused on gaze (or fixation), saccade, and tracking training $(44,45)$. Duration of intervention was derived from similar studies $(44,45)$. The control group continued onto conventional therapy at the learning disabilities center based on their previous treatment protocol. Rehabilitation exercises were designed and approved by experts in the field of balance in audiology groups. A brochure of the same exercises was designed for the parents and was taught to them and to the children to continue rehabilitation exercises at home, in addition to clinic exercises. Parents were told that homework had to be done 3 times a day for 15 minutes each time.

In saccade training, the child should sit in a comfortable position and put a card (or an image) in each hand at a distance of $45 \mathrm{~cm}$ in front of her/his eyes. Then, while keeping her/his head constant, eyes should be quickly moved from one card to another without stopping between cards. The child should be taught to move only her/his eyes. As the training progresses, the child is asked to concentrate on small components of cards (46).

In tracking training, the child should keep a small card in front of his/her eyes at a distance of $30 \mathrm{~cm}$ (a few words are written on cards), then, slowly move the card in the horizontal direction to the right and left and back to the center. During exercise, the child's head should be constant and only follow the card with the eyes. She/he should repeat this motion in the vertical and diagonal directions. To continue, the child is asked to move the arms fast and faster until he/she fails to read the words. The child should be repeating these exercises 15 to 20 times in horizontal, vertical, and diagonal directions (46).

In fixation training, the child should be sitting in a comfortable position (preferably on a chair), and she/he should consider 3 target points (for example, a clock, a picture, and a lamp) at the same level of her/his eyes. One of the target points should be placed on the top of the left shoulder, another in the front, and the other should be located on her/his right shoulder. The child should move the head to the left, center, and right targets, and then, should rotate the head 10 to 15 times without stopping on the targets. After finishing this step, the child should repeat the process again, focusing and stopping on the target for a few moments (46).

In the treatment phase, we provided a combination of repeated stimulation, horizontal, and vertical change of target position. We also increased the difficulty level of the target (for example, reducing the size of the stimulus), significantly increased attentional demand, and provided visual and verbal feedback. With the active participation of children in the study and their high motivation to do the tasks during the 8-week training course, the effect of rehabilitation was increased.

Statistical methods: Kolmogorov-Smirnov (K-S) test was used to evaluate the distribution of data. K$\mathrm{S}$ test showed that the distribution of data across all variables was normal except the saccade velocity variable. So, to analyze the results of this variable, corresponding nonparametric tests were used. Independent $t$ test (equivalent nonparametric test, Mann-Whitney test) and paired t test (equivalent nonparametric test, Wilcoxon signed 
ranks test) were used to compare the results between case and control groups and the results before and after the intervention in each group, respectively. To compare the results between the 3 groups (normal, case, and control), one-way ANOVA test (equivalent nonparametric test, Kruskal-Wallis test) was used. In addition, McNemar's test was used to analyze 4 qualitative variables (presence or absence of spontaneous nystagmus, gaze nystagmus and symmetry/dissymmetry of tracking, and optokinetic responses) before and after the intervention.Furthermore, In this study, repeated measures test was used to analyze intragroup and out-of-group interactions in pre- and post-intervention stages. Also, effect size and power were calculated. Data were analyzed using SPSS 21, and p-values less than 0.05 was considered significant for all tests.

\section{Results \\ Comparison of oculomotor results between dyslexic and non-dyslexic children}

Basic assessments of the 2 dyslexic and non-dyslexic children by oculomotor tests revealed very large differences between the 2 groups on these tests. Results are demonstrated in Table 3. Our basic findings showed that latency and velocity of saccade were more in dyslexic children and the accuracy of saccade was less than non-dyslexic children, indicating hypermetric eye movements in dyslexic children, so that the image of the target was faded and dark and was not complete on the retina. In addition, in tracking and optokinetic test, gain of response in dyslexic children was less than that of nondyslexic children, moreover, responses between the left and right eyes were asymmetric. Therefore, the child was not able to pursue the target correctly. Analysis of the average differences of these parameters showed significant differences between dyslexic and non-dyslexic children in all oculomotor tests $(\mathrm{p}<0.001)$.

Furthermore, the analysis of oculomotor results between the 3 groups (healthy, case, and control) showed significant differences in some of the oculomotor tests, but not in some others. We found significant differences in tracking gain $(\mathrm{p}<0.001)$, saccade latency $(\mathrm{p}<0.001)$, saccade accuracy $(p<0.001)$, and optokinetic gain $(p=0.050)$ variables, but no significant differences were observed in the saccade velocity variable. Table 4 demonstrates the oculomotor results between the 3 groups.

Evaluation of the effects of oculomotor rehabilitation therapy on recovery of eye movements in dyslexic children: Within- and between-group analyses

In this stage, after completion of rehabilitation exercises, case and control groups were retested by oculomotor tests. Figure 2 shows the comparison of the means in preand post- intervention stages within each group. Results of within group analysis revealed a significant difference between all oculomotor variables $(p<0.001)$ in the case group, except for optokinetic gain $(p=0.501)$ and saccade velocity $(p=0.161)$. In this group, the average of saccade accuracy increased from $70.62 \%$ to $90.92 \%$, and saccade latency decreased from $269.93 \mathrm{~ms}$ to 206.13 $\mathrm{ms}$ and fell in the normal range. Also, the mean of tracking gain increased from $72.34 \%$ to $84.03 \%$, and catch-up saccades decreased and reached to near those of the nondyslexic children.

There was no significant difference among oculomotor variables, and the values of all variables were almost the same in the control group $(\mathrm{p}>0.05)$. Between-group analyses were also performed. Results revealed no significant difference between case and control groups in any of the oculomotor variables before the intervention $(p>0.05)$. However, after the intervention, a significant difference was observed between the 2 groups in all oculomotor variables $(\mathrm{p}<0.001)$, except for optokinetic gain $(p=0.591)$.

The results of repeated measures test showed that had a significant effect on the tracking gain, and its effect was high $(p<0.001$, partial $\eta 2=0.99$, power $=1)$. The same effect was observed in saccade latency $(p<0.001$, partial $\eta 2=0.25$, power $=0.8)$ and saccade accuracy $(\mathrm{p}<0.001$, partial $\eta 2=0.53$, power $=1$ ), but oculomotor rehabilitation did not have any effect in saccade velocity $(\mathrm{p}=0.112$, partial $\eta 2=0.08$, power $=0.3)$, optokinetic gain $(p=0.513$, partial $\eta 2=0.01$, power $=0.09)$, and oculomotor intervention.

Table 3.The mean and standard deviation of the baseline oculomotor tests in dyslexic and non-dyslexic children

\begin{tabular}{lccc}
\hline Oculomotor variables & $\begin{array}{c}\text { Non-dyslexic children } \\
(\mathrm{N}=20)\end{array}$ & $\begin{array}{c}\text { Dyslexic children } \\
(\mathrm{N}=30)\end{array}$ & $\mathrm{p}$ \\
\hline Tracking gain $(\%)$ & $90.20(2.87)$ & $73.03(7.72)$ & $<0.001$ \\
Saccade latency (ms) & $205.46(25.68)$ & $264.82(25.64)$ & $<0.001$ \\
Saccade velocity (deg/sec) & $461.03(85.07)$ & $475.25(89.61)$ & $<0.001$ \\
Saccade accuracy (\%) & $91.30(8.13)$ & $70.66(7.33)$ & $<0.001$ \\
Optokinetic gain $(\%)$ & $90.72(11.33)$ & $78.57(20.10)$ & $<0.001$ \\
\hline
\end{tabular}

Table 4. The mean and standard deviation of comparison of oculomotor tests among the 3 groups of healthy, case, and control children in the pre-intervention stage

\begin{tabular}{lcccc}
\hline Oculomotor variables & Normal & Case & Control & $\mathrm{p}$ \\
& $\mathrm{N}=20$ & $\mathrm{~N}=15$ & $\mathrm{~N}=15$ & $<0.001$ \\
\hline Tracking gain $(\%)$ & $90.20(2.87)$ & $72.34(6.32)$ & $73.72(9.09)$ & $<0.001$ \\
Saccade latency $(\mathrm{ms})$ & $205.46(25.68)$ & $269.93(26.08)$ & $259.70(25.01)$ & 0.837 \\
Saccade velocity $(\mathrm{deg} / \mathrm{sec})$ & $461.03(85.07)$ & $465.48(81.25)$ & $485.02(99.15)$ & $<0.001$ \\
Saccade accuracy $(\%)$ & $91.30(8.13)$ & $70.62(7.53)$ & $70.70(7.38)$ & 0.053 \\
Optokinetic gain $(\%)$ & $90.72(11.33)$ & $76.79(17.50)$ & $80.35(22.89)$ & \\
\hline
\end{tabular}


A
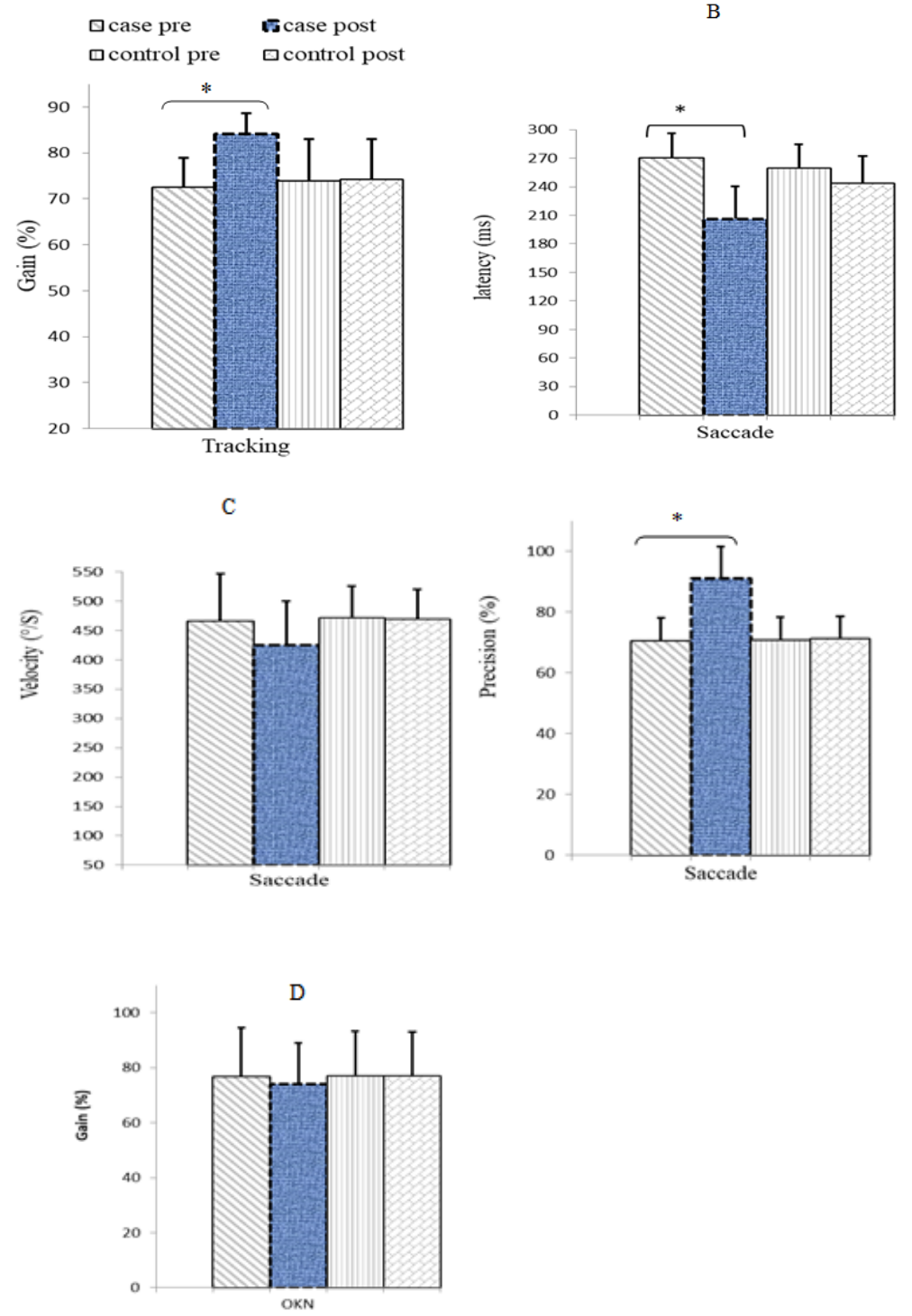

Fig. 2. The mean comparison of oculomotor responses before and after the intervention between the 2 groups of dyslexic children vertical lines indicate standard errors

Comparison of qualitative variables of oculomotor tests in the pre- and post- interventionstages

In this section, qualitative variables were compared in the study population in 2 stages (before and after the intervention). In the spontaneous nystagmus test, 22 children out of 50 had a normal response and 28 had an abnormal response. Repeating the test after the intervention showed that only 12 individuals from 28 patients still had spontaneous nystagmus; however, spontaneous nystagmus was not observed in 16 children after the intervention. Analysis of results by McNemar test showed that this effect (improvement of spontaneous nystagmus by intervention) was significant $(\mathrm{p}<0.001)$. Also, in the Gaze test, we found that 25 children had gaze nystagmus, which was corrected in 13 children and they were moved to the healthy group; and finally, 38 children had a normal response at the end of the intervention. In this study, 8 and 15 of 50 children had asymmetric tracking and optokinetic response, respectively. After the intervention, 7 and 14 children had more symmetric tracking and optokinetic response compared to before the intervention, respectively. Comparison of the results before and after the intervention showed the significant effect of the oculomotor intervention on the gaze nystagmus $(\mathrm{p}<0.001)$, symmetry of optokinetic response $(\mathrm{p}<0.001)$, and symmetry of tracking response $(\mathrm{p}<0.001)$.

\section{Discussion}

The results revealed that the oculomotor rehabilitation program improved most of eye movements in children with dyslexia, except for optokinetic response. Before the intervention, parents of dyslexic children complained of reading and visual search problems of their children and reported that reading had become a very difficult and frustrating activity for their children. These reports were in agreement with behavioral evaluation as well as eye movements recording, so that all dyslexic children showed significant disruption in the pattern of eye movements.

\section{$6 \quad$ http://mjiri.iums.ac.ir}


After the intervention, parents described children's reading to be much quicker, more fluent, and less effortful than the pre-intervention stage, syllables and words were omitted rarely and few mistakes were seen in reading. The subjective reports of parents were in close agreement with the treatment effects that were confirmed with objective findings of oculomotor tests, so that patterns of eye movement in all dyslexic children in the case group were improved. In this study, the role of visual attention in improving eye movements was not evaluated objectively, and only subjective reports of parents on the improvement of reading and visual attention of dyslexic children were reported. There are many similar studies in this area showing that individuals with both developmental (15-25) and acquired dyslexia $(14,16-18,47-56)$ have abnormal eye movements during reading.

Oculomotor deficits in dyslexic children could be due to immaturity of visual attentional strategies that leads to reduction in visual attentional span and reduction in visual information processing simultaneously. Bosse et al (57) found similar results, and some others have also confirmed these results (16-18). In addition, poor visual system in dyslexia was suggested for many years (57-60). In this regard, poor binocular coordination during prolonged fixations (61), visual confusion during reading (62), and poor eye alignment during fixation have been reported (8). Also, Iles et al. reported impairment in visual search with a motion deficit in dyslexic adults, which confirms the Magnocellular hypothesis of dyslexia (63). Results of the present study showed a dysfunction in the brainstem level and vestibular nuclei (The region involved in developing oculomotor responses.) in children with dyslexia, and this deficit causes bottom-up problems in children's reading ability. On the other hand, with respect to the role of top-down processes, it seems that visual attention processes have been involved in this as well. Overall, the findings suggest that perhaps both bottom-up and top-down processes are involved in the incidence of dyslexia disorders (16-18).

Although there have been reports of eye movements improvement following oculomotor training in people with acquired dyslexia(15-19), studies addressing children with developmental dyslexia are scarce. The findings of Schuett research, in line with findings of this study, showed that oculomotor rehabilitation intervention had positive effects in patients with mild traumatic brain injury (mTBI), who were suffering from oculomotor impairment and reading disabilities. In their study, treatment effects were characterized by an increase in reading speed and normalization of eye movements' pattern. They found that patients made fewer fixations with shorter duration after the intervention; they also indicated that saccadic amplitude increased and the number of forward saccades decreased. In addition, after treatment, patients were able to extract the same amount of text information using efficient oculomotor strategy and consuming less energy (14). A study by Burkhart Fischer et al. also demonstrated that daily practice of visual orientation detection tasks in fixation or saccade and/or distracter condition not only improves attention capacity but also improves saccadic behavior in the dyslexic children. Also, about a third of children showed improvement in reading ability, and some of them represented an immediate and significant improvement in handwriting, which was reported by parents or teachers (64). Similar results were obtained in studies by Galaburda and Eden $(65,66)$.

Similarly, in a study by Thiagarajan, following oculomotor rehabilitation therapy (OMT) in patients with mTBI, less effort was made to read, and marked increase in visual attention and reading speed was also observed (1). These findings suggest that following oculomotor rehabilitation therapy, general attention is assigned to the comprehension of text rather than engaging in low-level aspects of oculomotor control. Furthermore, oculomotor remediation per se is a part of the attentional training, and the results showed that visual attention was increased when using associated cognitive demands (e.g., continuous attention to target) along with visual feedback, which involves perceptual learning in the training process. These results generally represent the role of visual attention in oculomotor maturity and the deep relationship of visual attention and visual memory with cognitive strategies in the reading process. These findings were also demonstrated in studies by Ciuffreda et al. (2006) and Neera Kapoor (2004) in patients with mTBI. They observed that the implementation of eye movements training and stimulation of reading led to marked improvement in the patients' attention in many environmental conditions $(1,41)$. Furthermore, an fMRI study of Corbetta et al. supported this assumption that attention and oculomotor processes are deeply integrated at the high neural level (67). In another study, Culham, Cavanagh, and Kanwisher have observed the obvious activation of attentional response in the parietal regions, particularly the left superior parietal area and its deficiency in dyslexics, which reflects the relationship between oculomotor performance and reading fluency $(68,69)$. In addition, Iles et al. reported that Magnocellular deficits that involve the parietal cortex could also be related to visual attention skills in dyslexic children.

Improvement of eye movements and reading ability in dyslexic children cannot be attributed to spontaneous recovery of the visual field or spontaneous adaptation of the oculomotor system because none of the dyslexic children in the control group (without intervention) represented an obvious change in oculomotor response during retesting. Improvement of oculomotor responses was observed only in the case group (with intervention) after the intervention. Therefore, long-term improvement with stability in reading (at least for a period of 12 weeks) can be attributed to systematic effects of oculomotor exercises, so that the oculomotor rehabilitation facilitates oculomotor adaptation, decreases word identification impairment, and improves oculomotor control during the text processing. Furthermore, dyslexia does not improve with any type of voluntary eye movements and any kind of visual material, but it requires precise and systematic eye movements training to strengthen the eye muscles. This has been shown in studies that have evaluated the effects of pure oculomotor exercises on reading performance in pa- 


tients with macular degeneration due to
age (Seipleet al., 2005) and in patients who have reading problems with visual/ oculomotor origin after acquired brain injury (Ciuffredaet al., 2006)(1).

Although the present investigation was relatively comprehensive, there were some study limitations: First, follow-up was not performed. Follow-up duration should be at least 6 months to investigate the long-term effects of rehabilitation. Second, we used diagnosed dyslexic children and did not use a reading test to diagnose dyslexia; moreover, we did not categorize dyslexic children based on severity of disorder. Third, we did not compare the case and control groups with the healthy group at the end of study. However, the main purpose of this was to analyze the impact of rehabilitation intervention on reading ability. Lastly, children with weakness in academic achievement might have shown reading problems as well, but we did not control this factor. However, for ethical considerations, brochures of rehabilitation exercises were awarded to parents of dyslexic children in the control group, who did not receive oculomotor rehabilitation, to do the exercises at home after completion of study.

\section{Conclusion}

Although the neurobiological and theoretical aspects of dyslexia and its treatment are still unknown, analyzing eye movements in dyslexic children showed oculomotor deficits in these children; moreover, it indicated that daily exercises of eye movements improve basic (but not sufficient) visual mechanisms and lead to fluent reading. Moreover, the findings supported the notion that perhaps a link exists among visual attention, oculomotor readiness, and reading comprehension. Furthermore, the findings indicated that oculomotor rehabilitation, along with specific visual attention and reading training, can be useful tools to improve the range of visual attention and oculomotor skills in children with dyslexia. However, further research is needed to understand the relationship between visual attention and oculomotor deficits in dyslexic children.

\section{Acknowledgements}

We would like to thank learning disability centers (Tehran, Iran) for their cooperation in confirming the diagnosis of dyslexic children. We also extend our gratitude to Dr. Nima Rezazadeh for his support during data collection. Also, we are thankful to all participants and their parents. This study was part of a $\mathrm{PhD}$. dissertation project in audiology supported by Iran University of Medical Sciences.

\section{Conflict of Interests}

The authors declare that they have no competing interests.

\section{References}

1. Thiagarajan P. Oculomotor rehabilitation for reading dysfunction in mild traumatic brain injury: State University of New York; 2012:207274.

2. Shaywitz SE, Fletcher JM, Holahan JM, Shneider AE, Marchione KE, Stuebing KK, et al. Persistence of dyslexia: The Connecticut longitudinal study at adolescence. Pediatrics. 1999;104:1351-9.
3. Olitsky SE, Nelson LB. Reading disorders in children. Pediatr Clin North Am. 2003;50:213-224.

4. Boller F, Grafman J. Handbook of Neuropsychology: Disorders of visual behavior. The Times prss. 2000;411-420.

5. Siok WT, Spinks JA, Jin Z, Tan LH. Developmental dyslexia is characterized by the co-existence of visuospatial and phonological disorders in Chinese children. Curr Biol. 2009;19:890-2.

6. Baum A. Cambridge handbook of psychology, health and medicine. 1997:207-220

7. Facoetti A, Molteni M. The gradient of visual attention in developmental dyslexia. Neuropsychologia. 2001;39:352-7.

8. Eden GF, Stein JF, Wood HM, Wood FB. Differences in eye movements and reading problems in dyslexic and normal children. Vision research. 1994;34:1345-58.

9. Kevan A, Pammer K. Visual deficits in pre-readers at familial risk for dyslexia. Vision research. 2008;48:2835-9.

10. Quercia P, Feiss L, Michel C. Developmental dyslexia and vision. Clin Ophthalmol. 2013; 7:869.

11. Olulade OA, Napoliello EM, Eden GF. Abnormal visual motion processing is not a cause of dyslexia. Neuron. 2013;79:180-90.

12. Stein J. Dyslexia: the Role of Vision and Visual Attention. Curr Dev Disord Rep. 2014;1:267-80.

13. Facoetti A, Luisa Lorusso M, Paganoni P, Umilta C, Gastone Mascetti G. The role of visuospatial attention in developmental dyslexia: evidence from a rehabilitation study. Cognit Brain Res. 2003;15:154-64.

14. Schuett S, Heywood CA, Kentridge RW, Zihl J. Rehabilitation of hemianopic dyslexia: Are words necessary for re-learning oculomotor control? Brain. 2008;131:3156-68.

15. Gori S, Facoetti A. How the visual aspects can be crucial in reading acquisition: The intriguing case of crowding and developmental dyslexia. J Vision. 2015;15.

16.Bucci MP, Nassibi N, Gerard CL, Bui-Quoc E, Seassau M. Immaturity of the oculomotor saccade and vergence interaction in dyslexic children: evidence from a reading and visual search study. PloS one. 2012;7(3):334-58.

17.Jainta S, Kapoula Z. Dyslexic children are confronted with unstable binocular fixation while reading. PLoS One. 2011;6(4):e18694.

18. Seassau M, Gérard CL, Bui-Quoc E, Bucci MP. Binocular saccade coordination in reading and visual search: a developmental study in typical reader and dyslexic children. Front Integrat Neuroscience. 2014;8(85):1-11.

19.Pavlidis GT. Do eye movements hold the key to dyslexia? Neuropsychologia. 1981;19(1):57-64.

20.Rayner K. Do faulty eye movements cause dyslexia? Developmental Neuropsychology. 1985;1(1):3-15.

21.De Luca M, Di Pace E, Judica A, Spinelli D, Zoccolotti P. Eye movement patterns in linguistic and non-linguistic tasks in developmental surface dyslexia. Neuropsychologia. 1999;37(12):1407-20.

22.Li XH, Jing J, Zou XB, Huang X, Jin Y, Wang QX, et al. Picture perception in Chinese dyslexic children: an eye-movement study. Chin. Med. J. 2009;122(3):267.

23. Trauzettel-Klosinski S, Koitzsch AM, Dürrwächter U, Sokolov AN, Reinhard J, Klosinski G. Eye movements in German-speaking children with and without dyslexia when reading aloud. Acta Ophthalmologica. 2010;88(6):681-91.

24.Hatzidaki A, Gianneli M, Petrakis E, Makaronas N, Aslanides IM. Reading and visual processing in Greek dyslexic children: an eye-movement study. Dyslexia. 2011;17(1):85-104.

25. Hutzler F, Kronbichler M, Jacobs AM, Wimmer H. Perhaps correlational but not causal: No effect of dyslexic readers' magnocellular system on their eye movements during reading. Neuropsychologia. 2006;44(4):637-48.

26.Kirkby JA, Blythe HI, Drieghe D, Liversedge SP. Reading text increases binocular disparity in dyslexic children. PLoS One. 2011;6(11):271-5.

27. Simos PG, Fletcher JM, Bergman E, Breier JI, Foorman BR, Castillo EM, et al. Dyslexia-specific brain activation profile becomes normal following successful remedial training. Neurology. 2002;58:1203-13.

28. Richards TL, Corina D, Serafini S, Steury K, Echelard DR, Dager $\mathrm{SR}$, et al. Effects of a phonologically driven treatment for dyslexia on lactate levels measured by proton MR spectroscopic imaging. Am J Neuroradiol. 2000;21:916-22.

29. Temple E, Deutsch GK, Poldrack RA, Miller SL, Tallal P, Merzenich 
MM, et al. Neural deficits in children with dyslexia ameliorated by behavioral remediation: evidence from functional MRI. Proc Natl Acad Sci USA. 2003;100:2860-5.

30.Kujala T, Karma K, Ceponiene R, Belitz S, Turkkila P, Tervaniemi $M$, et al. Plastic neural changes and reading improvement caused by audiovisual training in reading-impaired children. Proc Natl Acad Sci USA. 2001;98:10509-14.

31.Russo NM, Nicol TG, Zecker SG, Hayes EA, Kraus N. Auditory training improves neural timing in the human brainstem. Behav. Brain Res. 2005;156:95-103.

32. Tierney AT, Kraus N, Merzenich MM, Nahum M, van Vleet $T$. Music training for the development of reading skills. Progr Brain Res. 2013;207:209-241.

33. Strait DL, Parbery-Clark A, Hittner E, Kraus N. Musical training during early childhood enhances the neural encoding of speech in noise. Brain and language. 2012;123:191-201.

34. Skoe E, Kraus N. Musical training heightens auditory brainstem function during sensitive periods in development. Front Psychol. 2013;4-11.

35.Harding JAH. The relationship between music and language achievement in early childhood. Front Psychol. 1989:12-13.

36.Paolozza A, Rasmussen C, Pei J, Hanlon-Dearman A, Nikkel SM, Andrew G, et al. Deficits in response inhibition correlate with oculomotor control in children with fetal alcohol spectrum disorder and prenatal alcohol exposure. Behav Brain Res. 2014;259:97-105.

37. Ball K, Pearson DG, Smith DT. Oculomotor involvement in spatial working memory is task-specific. Cognition. 2013;129:439-46.

38.Paolozza A, Rasmussen C, Pei J, Hanlon-Dearman A, Nikkel SM, Andrew G, et al. Working memory and visuospatial deficits correlate with oculomotor control in children with fetal alcohol spectrum disorder. Behav. Brain Res. 2014:263:70-9.

39. Han Y, Ciuffreda KJ, Kapoor N. Reading-related oculomotor testing and training protocols for acquired brain injury in humans. Brain Research. 2004;14(1):1-12.

40.Pouget MC, Le'vy-Bencheton D, Prost M, Tilikete C, Husain M, Jacquin-Courtois S. Acquired visual field defects rehabilitation: Critical review and perspectives. Annal Physical Rehab Med. 2012;55:53-74.

41.Kapoor N, Ciuffreda KJ, Han Y. Oculomotor Rehabilitation in Acquired Brain Injury: A Case Series. Arch Phys Med Rehabil. 2004;85:1667-78.

42. Kerkhoff G, Schenk T. Rehabilitation of neglect: An update. Neuropsychologia. 2012;50:1072- 9.

43. Shepard NT, Schubert M. Background and Technique of Ocular Motility Testing. In: Jacobson GP, Shepard NT, editors. Balance Function Assessment and Management. 1 ed. United Kingdom.2008. p. 133-71.

44.Desmond A. Vestibular function,evaluation and treatment. .The Times prss. 2004:77-88.

45. Gans RE. Vestibular Rehabilitation: Protocols and Programs. Otology \& Neurotology. 1997; 18:50-61.

46. Gans RE. Handbook of Vestibular Rehabilitation, Protocols and Programs. .The Times prss 1997:55-70.

47. Spitzyna G, Wise R, McDonald S, Plant G, Kidd D, Crewes H, et al Optokinetic therapy improves text reading in patients with hemianopic alexia A controlled trial. Neurology. 2007;68(22):192230.

48.De Luca M, Spinelli D, Zoccolotti P. Eye movement patterns in reading as a function of visual field defects and contrast sensitivity loss. Cortex. 1996;32(3):491-502.

49.Eber A, Metz-Lutz M, Bataillard M, Collard M. Reading eye movements of patients with homonymous hemianopia. Eye Move Physio Cog. 1987:544-5.

50.Kerkhoff G, Münßinger U, Eberle-Strauss G, Stögerer E. Rehabilitation of hemianopic alexia in patients with postgeniculate visual field disorders. Neuropsychological Rehabilitation. 1992;2(1):21-42.

51.Leff A, Scott S, Crewes H, Hodgson TL, Cowey A, Howard D, et al. Impaired reading in patients with right hemianopia. Annal Neurol. 2000;47(2):171-8.

52.McDonald SA, Spitsyna G, Shillcock RC, Wise RJ, Leff AP. Patients with hemianopic alexia adopt an inefficient eye movement strategy when reading text. Brain. 2006;129(1):158-67.

53. Schoepf D, Zangemeister WH. Correlation of ocular motor reading strategies to the status of adaptation in patients with hemianopic visual field defects. Annals of the New York Academy of Sciences. 1993;682(1):404-8.

54.Trauzettel-Klosinski S, Brendler K. Eye movements in reading with hemianopic field defects: the significance of clinical parameters. Graefe's Arch Clin Experi Ophthalmol. 1998;236(2):91-102.

55.Zihl J. Eye movement patterns in hemianopic dyslexia. Brain 1995;118(4):891-912.

56.Zihl J. Rehabilitation of visual disorders after brain injury: Psychology Press; 2010.

57.Bosse ML, Tainturier MJ, Valdois S. Developmental dyslexia: The visual attention span deficit hypothesis. Cognition. 2007;104(2):198230 .

58. Galaburda AM, Sherman GF, Rosen GD, Aboitiz F, Geschwind N. Developmental dyslexia: four consecutive patients with cortical anomalies. Annal Neurol. 1985;18(2):222-33

59. Peyrin C, Démonet J, N'Guyen-Morel M, Le Bas J, Valdois S. Superior parietal lobule dysfunction in a homogeneous group of dyslexic children with a visual attention span disorder. Brain Lang. 2011;118(3):128-38

60.Prado C, Dubois M, Valdois S. The eye movements of dyslexic children during reading and visual search: impact of the visual attention span. Vision Res. 2007;47(19):2521-30.

61.Stein J, Fowler M. Unstable binocular control in dyslexic children. Journal of Research in Reading. 1993;16(1):30-45.

62. Stein J, Walsh V. To see but not to read; the magnocellular theory of dyslexia. Trends Neurosciences. 1997;20(4):147-52.

63.Iles J, Walsh V, Richardson A. Visual search performance in dyslexia. Dyslexia. 2000;6(3):163-77.

64.Fischer B, Hartnegg K. Effects of visual training on saccade control in dyslexia. Perception. 2000;29:531-42.

65. Galaburda A, Livingstone M. Evidence for a Magnocellular Defect in Developmental Dyslexia a. Annal New York Acad Sci. 1993;682(1):70-82.

66.Eden GF, VanMeter JW, Rumsey JM, Maisog JM. Abnormal processing of visual motion in dyslexia revealed by functional brain imaging. Nature. 1996;382(6586):66.

67. Corbetta M, Akbudak E, Conturo TE, Snyder AZ, Ollinger JM, Drury $\mathrm{HA}$, et al. A common network of functional areas for attention and eye movements. Neuron. 1998;21(4):761-73.

68. Solan HA, Shelley-Tremblay J, Ficarra A, Silverman M, Larson S. Effect of attention therapy on reading comprehension. J Learn Disab. 2003;36(6):556-63.

69. Culham JC, Cavanagh P, Kanwisher NG. Attention response functions: characterizing brain areas using fMRI activation during parametric variations of attentional load. Neuron. 2001;32(4):737-45.

70. Warraich Z, Kleim JA. Neural plasticity: the biological substrate for neurorehabilitation. PM\&R. 2010;2(12):208-19. 